\title{
Sequência de Moebius e a condição de saúde bucal: Relato de caso
}

\section{Moebius sequence and the oral health condition: Case report}

\author{
Secuencia de Moebius y estado de salud bucal: Reporte de caso
}

Jeani Aparecida Petrik Brischke ORCID: https://orcid.org/0000-0002-6347-8242 Universidade Estadual do Oeste do Paraná, Brasil E-mail: jeanipetrik1@gmail.com

Célia Patrícia Müller Rodrigues ORCID: https://orcid.org/0000-0002-7752-0560 Universidade Estadual do Oeste do Paraná, Brasi E-mail: pattymiiller@hotmail.com

Juliana Marques Lindenberg ORCID: https://orcid.org/0000-0002-2413-9475 Universidade Estadual do Oeste do Paraná, Brasi E-mail: marquesjuli@yahoo.com.br Carlos Augusto Nassar ORCID: https://orcid.org/0000-0002-8647-413X Universidade Estadual do Oeste do Paraná, Brasil E-mail: carlos.nassar@unioeste.br

Mariângela Monteiro de Melo Baltazar ORCID: https://orcid.org/0000-0001-6395-9516 Universidade Estadual do Oeste do Paraná, Brasil E-mail: mmmwgb@uol.com.br

\begin{abstract}
Resumo
A Síndrome de Moebius é uma desordem congênita neurológica rara, com prevalência de 1:250.000 indivíduos, sem etiologia definida. A principal característica da síndrome é a paralisia do nervo facial (VII par de nervo craniano), podendo comprometer outros nervos, o diagnóstico pode ser dado ainda na infância. $\mathrm{O}$ tratamento deve ser realizado por uma equipe multiprofissional com acompanhamento periódico. O objetivo desse trabalho foi relatar o caso de uma paciente acometida pela Síndrome de Moebius dando ênfase à condição de saúde bucal. Este trabalho foi realizado no Centro de Atenção e Pesquisa em Anomalias Craniofaciais - CEAPAC localizado na cidade de Cascavel- PR. Paciente M. C. S. R. com dezenove anos de idade, sexo feminino, foi encaminhada ao CEAPAC para realização de tratamento odontológico. Ao exame físico observou-se características típicas de portadores da Síndrome de Moebius e o exame intraoral revelou precária condição dentária e dos tecidos adjacentes. O tratamento odontológico foi realizado em ambiente clínico e sob anestesia local. Considerando as graves implicações desencadeadas pela síndrome é de extrema importância o acompanhamento do paciente por uma equipe multiprofissional de maneira precoce e periódica.
\end{abstract}

Palavras-chave: Síndrome de Möbius; Odontologia; Paralisia facial; Assistência odontológica para pessoas com deficiências.

\begin{abstract}
Moebius Syndrome is a rare congenital neurological disorder, with a prevalence of 1:250,000 individuals, with no defined etiology. The main feature of the syndrome is facial nerve palsy (VII pair of cranial nerve), which can compromise other nerves, the diagnosis can be made in childhood. Treatment must be carried out by a multidisciplinary team with periodic monitoring. The objective of this work was to report the case of a patient affected by Moebius Syndrome, emphasizing the oral health condition. This work was carried out at the Center for Attention and Research in Craniofacial Anomalies - CEAPAC located in the city of Cascavel-PR. Patient M.C.S.R. nineteen years old, female, was referred to CEAPAC for dental treatment. On physical examination, typical characteristics of patients with Moebius Syndrome were observed, and the intraoral examination revealed a precarious condition of the teeth and adjacent tissues. Dental treatment was performed in a clinical setting and under local anesthesia. Considering the serious implications triggered by the syndrome, early and periodic monitoring of the patient by a multidisciplinary team is extremely important.
\end{abstract}

Keywords: Mobius syndrome; Dentistry; Facial paralysis; Dental care for disabled.

\section{Resumen}

El síndrome de Moebius es un trastorno neurológico congénito poco común, con una prevalencia de 1: 250.000 individuos, sin etiología definida. La característica principal del síndrome es la parálisis del nervio facial (VII par de 
pares craneales), que puede comprometer otros nervios, el diagnóstico se puede hacer en la infancia. El tratamiento debe ser realizado por un equipo multidisciplinar con seguimiento periódico. El objetivo de este trabajo fue reportar el caso de un paciente afectado por el Síndrome de Moebius, enfatizando la condición de salud bucal. Este trabajo se llevó a cabo en el Centro de Atención e Investigación en Anomalías Craneofaciales - CEAPAC ubicado en la ciudad de Cascavel-PR. La paciente M.C.S.R. de diecinueve años, mujer, fue remitida a CEAPAC para tratamiento dental. El examen físico mostró características típicas de los pacientes con Síndrome de Moebius y el examen intraoral reveló un estado precario de los dientes y tejidos adyacentes. El tratamiento dental se realizó en un entorno clínico y bajo anestesia local. Teniendo en cuenta las graves implicaciones que desencadena el síndrome, es de suma importancia el seguimiento precoz y periódico del paciente por parte de un equipo multidisciplinar.

Palabras clave: Síndrome de Mobius; Odontología; Parálisis facial; Atención dental para personas con discapacidades.

\section{Introdução}

A Síndrome de Moebius (SM), descrita por Von Graefe em 1880, é uma desordem congênita neurológica rara não progressiva de severidade variada. A prevalência está estimada em 1:250.000 atingindo ambos os sexos da mesma forma, sendo que apenas $2 \%$ dos casos documentados apresentam antecedentes familiares (Nagaraj, et al, 2020). A etiologia não é clara, no entanto, a hipótese mais aceita é da isquemia das células nervosas do embrião, durante os 3 primeiros meses de gestação, provocada pela parada do fluxo sanguíneo que pode ser decorrente do uso de substâncias tóxicas ou medicamentos. Rubéola gestacional, diabetes, aumento da temperatura corporal, distúrbio no desenvolvimento vascular romboencefálico (causado por alterações genéticas e relações consanguíneas) são outras possíveis causas. A síndrome pode ser identificada ainda no período infantil a partir da observação do incompleto fechamento da pálpebra durante o sono, falta de sorriso e mímica facial e dificuldade de sucção (Soares \& Pinchemel, 2018).

A principal característica da síndrome é a paralisia do nervo facial (VII par de nervo craniano) geralmente bilateral, por essa razão os indivíduos apresentam a face em máscara. O nervo abducente também pode ser afetado e é possível que haja malformação das extremidades superiores e inferiores e no sistema límbico (responsável por emoções e comportamentos sociais) (Pedersen, et al, 2017). Alterações orofaciais também podem ser encontradas, tais como: fissura palatina, língua fissurada, deficiência de tônus dos músculos faciais e da língua, hipoplasia dentária, cárie, doença periodontal, micrognatia, microstomia, mordida aberta, úvula bífida, dificuldade em realizar movimentos excursivos com a mandíbula, pequenas fissuras palpebrais, ptose palpebral, epicanto bilateral, hipertelorismo ocular, deformidade da orelha externa com perda ocasional da audição (Serra, et al, 2017).

O tratamento deve ser realizado por uma equipe multiprofissional com acompanhamento periódico, tendo em vista que a síndrome afeta consideravelmente a vida do indivíduo e seus familiares, podendo gerar desordens psicológicas, além das alterações funcionais (Ciupa, et al, 2021). No que compete a odontologia é indispensável que o paciente seja acompanhado o mais precocemente possível, a fim de prevenir doenças, como a cárie e doença periodontal, que quando não tratadas podem gerar sérias complicações (Melo, et al,2020).

Diante disso, o objetivo desse trabalho foi relatar o caso de uma paciente acometida pela Sequência de Moebius dando ênfase à condição de saúde bucal e plano de tratamento realizado pelo setor de odontologia do Centro de Atenção e Pesquisa em Anomalias Craniofaciais - CEAPAC.

\section{Metodologia}

Trata-se de um estudo de caso, realizado por meio de entrevista com a paciente e sua genitora e por observação direta. Os dados foram coletados no CEAPAC anexo ao Hospital Universitário do Oeste do Paraná (HUOP) na cidade de CascavelPR, a partir da anamnese e exame clínico. O estudo seguiu os preceitos éticos sendo aprovado previamente pelo comitê de 
ética em pesquisa sob o parecer $\mathrm{n}^{\circ} 4.250 .143$ e com o consentimento da paciente e sua genitora as quais assinaram o Termo de Consentimento Livre e esclarecido e autorizaram o uso de imagem.

\section{Relato de Caso}

Paciente M. C. S. R com dezenove anos de idade, diagnosticada com Sequência de Moebius, foi encaminhada pelo seu município de origem para o Centro de Atenção e Pesquisa em Anomalias Craniofaciais - CEAPAC, localizado em Cascavel-PR, para avaliação da equipe.

A paciente estava acompanhada da mãe, a qual relatou ter procurado atendimento odontológico para a filha no município de origem, porém os profissionais que as atenderam alegaram que não teriam condições de tratá-la e que a mesma deveria ser atendida em ambiente hospitalar.

A paciente foi avaliada pela equipe multiprofissional do CEAPAC a qual constatou as seguintes alterações: paralisia facial bilateral, pé direito valgo, estrabismo, olhos ressecados e ausência de selamento labial como mostrado na Figura 1 A, B, C.

Figura 1. Paciente com Sequência de Moebius onde é possível visualizar as características faciais: lábios sem selamento, estrabismo e aspecto da face em máscara (A) e pé direito valgo (B, C).

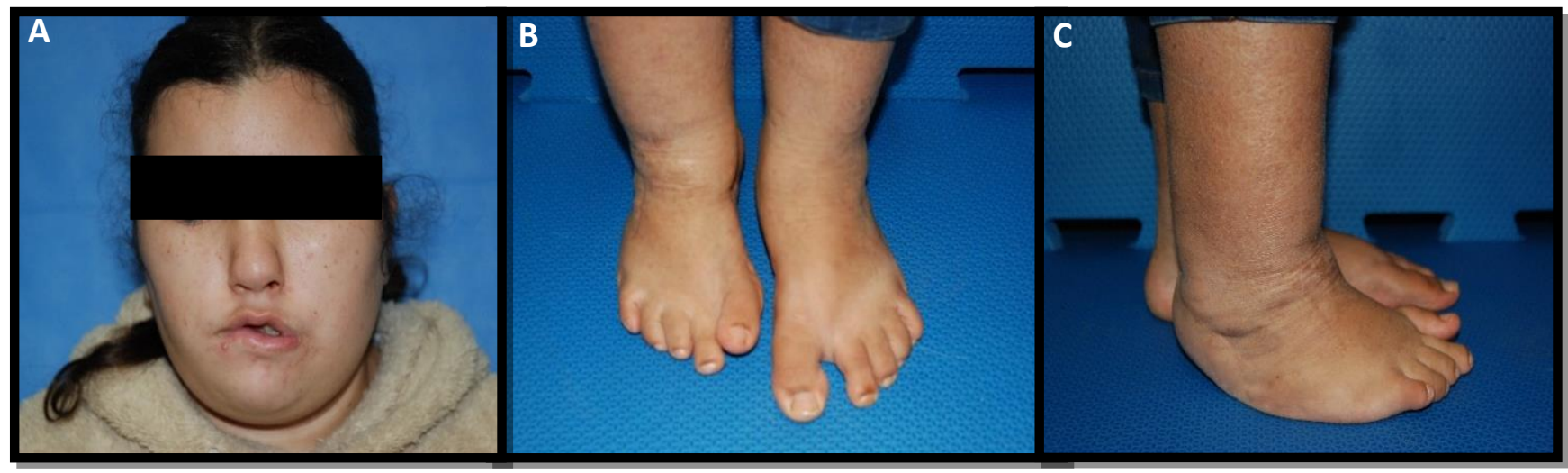

Fonte: Autores.

No exame intraoral evidencia-se má higiene bucal, presença de biofilme espesso, hipoplasia de esmalte, raízes residuais, cárie, gengivite, má oclusão, palato ogival, língua hipotônica de volume aumentado com pouca mobilidade e presença de fasciculações como é possível visualizar na Figura 2 A, B, C, entretanto na anamnese, relatou não possuir doenças sistêmicas.

Após exame clínico foi elaborado o plano de tratamento odontológico que consistiu em: exodontia das raízes residuais, a fim de cessar pontos de infecção; orientação de higiene e tratamento periodontal, para melhorar a saúde bucal evitando que a condição se agrave; restaurações necessárias e uma prótese dentária superior para amenizar impactos decorrente das perdas dentárias que é observada na figura 2 D e 2 E. A prótese inferior não foi confeccionada devido a falta de espaço interoclusal, entretanto a equipe está buscando alternativas para reabilitar a arcada inferior da paciente. Paciente foi encaminhada para avaliação ortodôntica devido à má oclusão severa, porém o tratamento não poderá ser realizado devido à ausência de dentes para ancoragem. O tratamento deste caso foi realizado em ambiente clínico sob anestesia local, quando houve necessidade, além de um trabalho motivacional e educativo interdisciplinarmente entre as especialidades. 
Figura 2. Fotos intraorais nas quais observa-se biofilme espesso, gengivite, língua fasciculada (B) e raízes residuais (A, B, C). Prótese superior instalada (D) e prótese fora da boca (E).

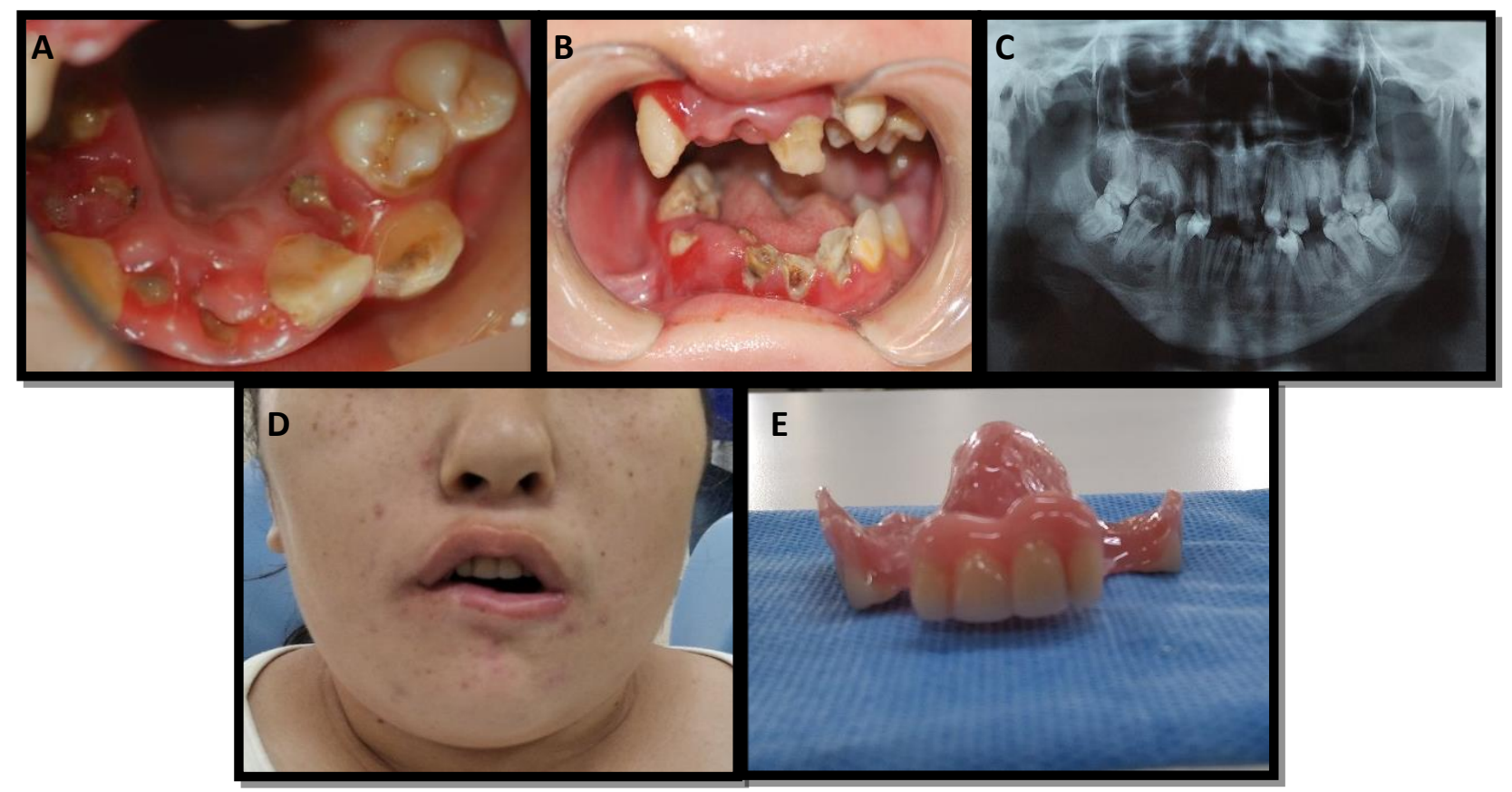

Fonte: Autores.

Retornos periódicos com a equipe de odontologia, serão necessários, para verificar a adaptação da prótese e se as instruções quanto à higiene bucal apresentaram bons resultados.

No intuito de garantir um atendimento multiprofissional, a paciente passou por atendimento com os demais profissionais do serviço e será acompanhada periodicamente pelas equipes: médica, de enfermagem, genética, psicologia, serviço social, fonoaudiologia, nutrição e fisioterapia.

\section{Resultados e Discussão}

As alterações apresentadas pela paciente como: paralisia bilateral do nervo facial, não selamento dos lábios, estrabismo, olhos ressecados e pé valgo, são características geralmente encontradas em pessoas com a Sequência de Moebius segundo a literatura pesquisada (Pedro, 2019; Saavedra, et al, 2018; Hoyle \& Durey, 2017; Mederos-Mollineda, et al, 2017).

(Mussi, et al, 2016) relata em seu trabalho que pessoas com Sequência de Moebius apresentam fluxo salivar diminuído e sua composição alterada fazendo com que a capacidade tampão seja menos eficaz, favorecendo o surgimento de cárie e doença periodontal. Esses fatores foram observados no caso relatado e sem dúvidas, contribuíram para o elevado número de lesões de cárie encontradas, além disso, constatou-se também a presença de hipoplasia de esmalte, alteração recorrente em pacientes com a Sequência de Moebius e hipomobilidade da língua que somadas dificultam a higiene e propiciam a instalação de biofilme (Soares \& Pinchemel, 2018).

Em casos, nos quais o paciente apresente limitação de abertura bucal, episódios de náusea e comportamento não colaborativo, como o relatado por (Filho, et al, 2020), há a necessidade de realizar os procedimentos em centro cirúrgico sob anestesia geral, contudo, tendo em vista que a paciente não possui limitações que impeçam as abordagens, o tratamento foi realizado inteiramente em ambiente clínico e sob anestesia local, porém houve dificuldade em realizar os procedimentos 
devido a abertura bucal limitada que a paciente apresentava, esta limitação é comum em pacientes com a Síndrome de Moebius (Santos, et al, 2021). Ademais, (Soares \& Pinchemel, 2018), aconselham a utilização de vaselina, a fim de diminuir o desconforto e evitar lesões nos lábios desses pacientes que sofrem com o ressecamento.

Malformações esqueléticas (classe II e III) são frequentemente encontradas em pacientes com Moebius, além de problemas oclusais como: mordida aberta anterior e mordida profunda (Cudzilo \& Matthews-Brzozowska, 2019; Del Vecchio \& Chávez, 2016), a paciente relatada neste trabalho apresentava alteração classe II e também mordida profunda, no entanto, devido a necessidade de realizar extrações dos dentes que estavam em situação de raiz residual e a falta de estrutura para ancoragem impossibilitou a realização do tratamento ortodôntico.

(Hoyle \& Durey, 2017) relatam dificuldades na reabilitação de pacientes com Moebius utilizando próteses removíveis e apontam a paralisia dos nervos facial e trigêmeo, a anatomia anormal da crista alveolar e anormalidades na forma e mobilidade da língua como possíveis impedimentos. Não foi possível confeccionar prótese parcial removível convencional para reabilitar a paciente, devido à falta de espaço interoclusal e a má oclusão severa, dificultando a adaptação da estrutura metálica, de forma que se optou por reabilitá-la com uma prótese do tipo removível provisória superior apenas.

O acompanhamento precoce e periódico desses pacientes é de suma importância, levando em conta que a Sequência de Moebius pode comprometer a capacidade motora, cognitiva e nutricional que ocasiona o surgimento de patologias orais (Serra, et al 2017; Soares \& Pinchemel, 2018). A paciente relatada nesse trabalho apresentava um quadro grave de saúde bucal, além de sobrepeso, dificuldades motora e de fala que poderiam ter sido evitados ou amenizados com atendimento precoce e acompanhamento periódico.

(Ciupa, et al, 2021; Soares \& Pinchemel, 2018; Del Vecchio \& Chávez, 2016), relatam em seus trabalhos dificuldade em encontrar literatura a respeito do manejo odontológico em pacientes com a síndrome de Moebius, essa mesma dificuldade foi sentida ao realizar esse trabalho, os autores citados relatam ainda que trabalhos sobre o assunto são de extrema relevância uma vez que a doença afeta estruturas importantes da face podendo ainda causar sérios problemas de autoestima, logo as informações podem auxiliar profissionais no diagnóstico e tratamento precoce e consequentemente melhorar a condição de vida do paciente e seus familiares.

A literatura pesquisada aponta a necessidade da realização do tratamento de pacientes com Síndrome de Moebius por uma equipe multidisciplinar e de maneira precoce, por isso é preciso que o diagnóstico seja feito corretamente (Pedersen, et al, 2017; Hoyle \& Durey, 2017; Del Vechio \& Chávez, 2016). A paciente deste relato chegou para atendimento tardiamente apresentando comprometimento odontológico grave, além de apresentar problemas na fala e marcha. Coube a equipe multiprofissional trabalhar primeiramente na reabilitação da paciente. Ressalta-se que o tratamento realizado não foi o ideal, entretanto, considerando o atraso para iniciar o tratamento e as limitações da paciente e do centro, o melhor tratamento foi oferecido e a paciente continuará a ser acompanhada pela equipe e se necessário passará por novas intervenções no intuito de melhorar sua saúde física e mental.

\section{Considerações Finais}

Considerando as graves implicações que a Sequência de Moebius pode gerar, é primordial o acompanhamento do paciente por uma equipe multiprofissional de maneira precoce e periódica, com a finalidade de amenizar ou evitar danos. Foi possível realizar o tratamento odontológico da paciente em ambiente clínico sob anestesia local, apesar de existir dificuldades para sua realização. A literatura que trata a respeito do tratamento odontológico de pacientes com Sequência de Moebius é escasso, evidenciando a necessidade de novas produções. 


\section{Referências}

Amaral, I. C. C. do. (2015). Síndrome de Moebius associada ao uso de misoprostol: relato de caso. Revista incelências. 5(1). https://revistas.cesmac.edu.br/index.php/incelencias/article/view/443.

Bartzela, T. N., Carels, C., \& Maltha, J. C. (2017). Update on 13 Syndromes Affecting Craniofacial and Dental Structures. Frontiers in physiology, 8, 1038. https://doi.org/10.3389/fphys.2017.01038.

Bell, C; Nevitt, S; Mckay, V.H \& Fattah A.Y (2019). Will the real Moebius syndrome please stand up? A systematic review of the literature and statistical cluster analysis of clinical features. Am J Med Genet. 179 (2), 257-265. https://pubmed.ncbi.nlm.nih.gov/30556292/

Brandão, B. C.; Queiroz, M. A. de; Filho, N. P.; Brandão, B. R.; Silva, G. de P. F. W. \& Díaz, S. K. V. (2008). Aspectos dentários em paciente com síndrome de Moebius: Relato de caso. Acta Odontológica Venezolana,46 (3), 319-322. http://ve.scielo.org/scielo.php?script=sci_arttext\&pid=S0001$63652008000300015 \& \operatorname{lng}=$ es\&tlng=es.

Castro, J. P., Vouga, L. A., Jorge, I. S., Cunha, M. P., Amorim, R., \& Palhau, L. (2021). Moebius syndrome - improving outcome with combined state-of-theart surgical and rehabilitation treatments. Nascer e crescer - birth and growth medical journal, 30(2), 105-108. https://doi.org/10.25753/BirthGrowthMJ.v30.i2.18911.

Ciupa, K. G. C; Bachour, J. A. \& Felipe, L. C. Da S. (2021). Síndrome de Moebius: Uma Revisão de Literatura, JNT- Facit Business and Technology Journal. 1(26), 212-225. http://revistas.faculdadefacit.edu.br/index.php/JNT/article/view/998.

Cudzilo, D., \& Matthews-Brzozowska, T. (2019). Moebius syndrome: The challenge of dental management. European journal of paediatric dentistry, 20(2), 143-146. https://doi.org/10.23804/ejpd.2019.20.02.12.

Pellegrin, M., Marcucci, L., Brogioni, L., \& Prati, G. (2021). Surgical Treatment of Clubfoot in Children with Moebius Syndrome. Children (Basel, Switzerland), 8(4), 310. https://doi.org/10.3390/children8040310.

Stefani, E., Nicolini, Y., Belluardo, M., \& Ferrari, P. F. (2019). Congenital facial palsy and emotion processing: The case of Moebius syndrome. Genes, brain, and behavior, 18(1), e12548. https://doi.org/10.1111/gbb.12548.

Vecchio, R. M. S. \& Chávez, M. C. M. (2016). Manejo Odontológico del Síndrome de Moebius. Revista de Odontopediatria Latinoamericana, 6 (2), 118-125. https://doi.org/10.47990/alop.v6i2.120.

Hoyle, P. \& Durey, K. (2017). Moebius syndrome. Oral Surgery, 10 (4), 235-238. https://onlinelibrary.wiley.com/doi/full/10.1111/ors.12251.

Mahrous, A., \& Thalji, G. (2018). Prosthodontic Management of a Patient with Moebius Syndrome: A Clinical Report. Journal of prosthodontics: official journal of the American College of Prosthodontists, 27(3), 299-305. https://doi.org/10.1111/jopr.12664.

McClure, P., Kilinc, E., Oishi, S., I Riccio, A., \& A Karol, L. (2017). Mobius Syndrome: A 35-Year Single Institution Experience. Journal of pediatric orthopedics, 37(7), e446-e449. https://doi.org/10.1097/BPO.0000000000001009.

McKay, V. H., Touil, L. L., Jenkins, D., \& Fattah, A. Y. (2016). Managing the child with a diagnosis of Moebius syndrome: more than meets the eye. Archives of disease in childhood, 101(9), 843-846. https://doi.org/10.1136/archdischild-2015-310043.

Mederos-Mollineda, K.; Fernández-Vélez, Y.; Escariz-Borrego, L.; Chávez-Guerra, V.; Díaz-Rodríguez, J.; Guadalupe-Vargas, M. (2017). Síndrome de Moebius: relato de caso. Revista Neurologia Argentina, 9(2), 90-95. https://www.elsevier.es/es-revista-neurologia-argentina-301-articulo-sindrome-moebiusreporte-un-caso-S1853002817300150

Melo, I. A; Silva, T. A; Sousa, A. A; Maurício, S. C. M; Castro, C. M. L; Donato, L. P. L; Antunes, R. S. P; Almeida, A. S (2020). A importância clínica das alterações orofaciais dos portadores da síndrome de Moebius: Revisão da literatura. Braz. J. Of Develop. 6(11), 85057 - 85062. https://doi.org/10.34117/bjdv6n11-064.

Mussi, M. M. C., Moffa, E., Castro, T., Lira Ortega, A., Freitas, G., Braga, M., Siqueira, W. L., \& Cury Gallottini, M. H. (2016). Salivary parameters and oral health in the Moebius syndrome. Special care in dentistry: official publication of the American Association of Hospital Dentists, the Academy of Dentistry for the Handicapped, and the American Society for Geriatric Dentistry, 36(5), 265-270. https://doi.org/10.1111/scd.12175.

Nagaraj, T.; Kongbrailatpam, S.; Pai, S. A.; Debnath, P.; Ramasamy, P. \& Mumtaz, S. (2020). Moebius syndrome: A review of literature. Journal of Advanced Clinical \& Research Insights. 7 (2), 25-29. http://dx.doi.org/10.15713/ins.jcri.292.

Pamplona, M., Ysunza, P. A., Telich-Tarriba, J., Chávez-Serna, E., Villate-Escobar, P., Sterling, M., \& Cardenas-Mejia, A. (2020). Diagnosis and treatment of speech disorders in children with Moebius syndrome. International journal of pediatric otorhinolaryngology, 138 , 110316. https://doi.org/10.1016/j.ijporl.2020.110316.

Picciolini, O., Porro, M., Cattaneo, E., Castelletti, S., Masera, G., Mosca, F., \& Bedeschi, M. F. (2016). Moebius syndrome: clinical features, diagnosis, management and early intervention. Italian journal of pediatrics, 42(1), 56. https://doi.org/10.1186/s13052-016-0256-5.

Pedersen, L. K.; Maimburg, R. D.; Hertz, J. M.; Gjorup, H.; Pedersen, T. K.; Madsen, B. M. \& Ostergaard, J. R (2017). Moebius sequence - A multidisciplinary clinical approach. Orphanet Journal of Rare Diseases. 12 (4), 2-11. https://doi.org/10.1186/s13023-016-0559-z.

Pedro, A. C. C (2019). Impacto do comprometimento motor oral e de condições sistêmicas na qualidade de vida relacionada à saúde de crianças e adolescentes com sequência de Moebius e síndrome de Down. Dissertação de mestrado em Ciências Odontológicas. Faculdade de odontologia da Cidade de São Paulo, São Paulo, SP, BR. https://docs.bvsalud.org/biblioref/2020/01/1048915/anacarolinacorazzapedroversaooriginal.pdf.

Saavedra, V. C; García, R. S; Meritú, A. S (2018). Síndrome de Moebius. Reporte de un caso clínico. Rev Mex Pediatr. 85 (3), $102-105$. https://www.medigraphic.com/cgi-bin/new/resumen.cgi?IDARTICULO=80803. 
Research, Society and Development, v. 10, n. 17, e120101724182, 2021

(CC BY 4.0) | ISSN 2525-3409 | DOI: http://dx.doi.org/10.33448/rsd-v10i17.24182

Serra, A. V. P; Moreira, C. V. A; Azevedo, R. A; Santos, N. D; Silva, L. O. R (2017). Síndrome de Moebius em paciente com fissura labiopalatina: Relato de caso. Revista Bahiana de Odontologia. 8 (4), 125-131. http://dx.doi.org/10.17267/2238-2720revbahianaodonto.v8i4.1562.

Santos, P., Lima, R., Santana, W., \& Gutierrez, G. (2021). Manifestações orofaciais da síndrome de Moebius: relato de caso. Jornada odontológica Dos acadêmicos Da católica, 6. Recuperado de http://reservas.fcrs.edu.br/index.php/joac/article/view/4527.

Soares, F.S; Pinchemel, E. N. B (2018). O impacto da síndrome de Moebius na saúde oral. Id on Line Rev. Mult. Psic. 12 (42), 66-74. https://doi.org/10.14295/idonline.v12i42.1415.

Romero, J. D., Buriticá, J. D. C., Paredes, A. I. (2017). Reporte de cuatro casos y revisión de literatura Síndrome de Moebiu s. Revista Colombiana Salud Libre, 12(2), 60-66. https://revistas.unilibre.edu.co/index.php/rcslibre/article/view/1737.

Telich-Tarriba, J. E., Navarro-Barquin, D. F., Verdezoto-Gaibor, K., \& Cardenas-Mejia, A. (2021). Prevalence of Hand Malformations in Patients With Moebius Syndrome and Their Management. Hand (New York, N.Y.), 1558944721994265. Advance online publication. https://doi.org/10.1177/1558944721994265.

Telegrafi, A., Webb, B. D., Robbins, S. M., Speck-Martins, C. E., fitzpatrick, D., Fleming, L. \& Sobreira, N. L. (2017). Identification of STAC3 variants in non-Native American families with overlapping features of Carey-Fineman-Ziter syndrome and Moebius syndrome. American Journal of Medical Genetics Part A, 173(10), 2763-2771. https://pubmed.ncbi.nlm.nih.gov/28777491/. 